\title{
PORRESPONDENCE
}

\section{Diabetes in Childhood and Adolescence}

A Guideline-Based Approach to Diagnosis, Treatment, and Follow-Up

by Prof. Dr. med. Andreas Neu and Dr. med. Ralph Ziegler in issue 9/2018

\section{Additional Comments}

To help prevent clinically relevant ketosis/ketoacidosis, ketone urine testing strips should be part of the basic equipment of any child with type 1 diabetes and their families. It is important to remember that the treatment of (severe) hypoglycemia should be initiated directly on site even if emergency admission is indicated. To prevent hypoglycemia it may be helpful to inform additional key contact persons and carers in advance about potential problems (travel companions, sports trainers, and friends/partners if the patient is an adolescent).

Psychosocial care as mentioned by the authors, needs to take into account that puberty and adolescence is a vulnerable period that entails risks. In this phase, adjustment disorders occasionally manifest as dysfunctional coping strategies, with severe effects on blood glucose control. Treating patients with such conditions requires much patience and competence. In our own setting, we found co-treatment by a psychodiabetologist optimally helpful. Group education sessions focusing on specific subjects can also be beneficial in this context, for example, contraception, handling intoxicating drugs (especially alcohol), pursuit of extreme sports, or weight management.

Insulin pumps and CGM systems, especially those providing hypoglycemia alerts, have enabled impressive progress in diabetes therapy. However, it should be kept in mind that the indi- cations for insulin pump treatment as explained in Box 1 of the article only rarely apply per se-for example, if a dawn phenomenon cannot be compensated for. Impaired quality of life as sole indication requires critical individual consideration. Decisive are rather constellations that include several of the listed problems - for example, unacceptable glycemic fluctuations during the day, accompanied by frequent nocturnal hypoglycemia. Appropriate framework conditions are essential—such as, in younger children, the long-term handling of such demanding therapeutic situations by the parents.

The authors' comment about the importance of practical and experiential pedagogical approaches in pediatric diabetes education is to be endorsed, in particular with reference to holiday camps or rehabilitation measures in institutions providing the relevant qualifications and expertise. For younger patients, rehabilitation measures including mother and child may be a recommendation, as long as the rehabilitation center provides a certified diabetes team.

DOI: 10.3238/arztebl.2018.0353a

\section{References \\ 1. Ziegler R, Neu A: Diabetes in childhood and adolescence-a guideline-based approach to diagnosis, treatment, and follow-up. Dtsch Arztebl Int 2018; 115 : $146-56$.}

\section{Prof. Dr. med. Uwe Fischer \\ Zinnowitz}

uwe.fischer01@t-online.de

\section{Conflict of interest statement}

The author declares that no conflict of interest exists.

The authors of the article have chosen not to publish a reply.

\section{-O CLINICAL SNAPSHOT}

\section{Cullen's Sign in Necrotizing Pancreatitis}

A 29-year-old man was referred to the hospital because of progressively severe pain of 4 days' duration that had begun in the epigastrium and then spread to the umbilical region and the entire abdomen. Clinical examination revealed a tensely distended abdomen with prominent brown discoloration of the skin around the umbilicus. The laboratory tests and an ultrasonographic examination confirmed the suspected diagnosis of acute (idiopathic) pancreatitis. The patient's further course was marked by subtotal pancreatic necrosis, splenic vein thrombosis, and the development of diabetes mellitus due to loss of pancreatic tissue (type $3 \mathrm{c}$ ). After three weeks of conservative treatment, first in an intensive care unit and then on the regular ward, the skin changes had regressed and the patient had recovered to some extent. Brownish-blue spots (ecchymoses) around the umbilicus are known as Cullen's sign and are considered to indicate an unfavorable prognosis in acute pancreatitis. They arise by the spread of pancreatic enzymes along the round ligament of the liver into the periumbilical fat, causing hemorrhagic necrosis. Cullen's sign and the analogous Grey Turner's sign of the flank are seen in only ca. $1 \%$ of patients with pancreatitis but are associated with up to $40 \%$ mortality.

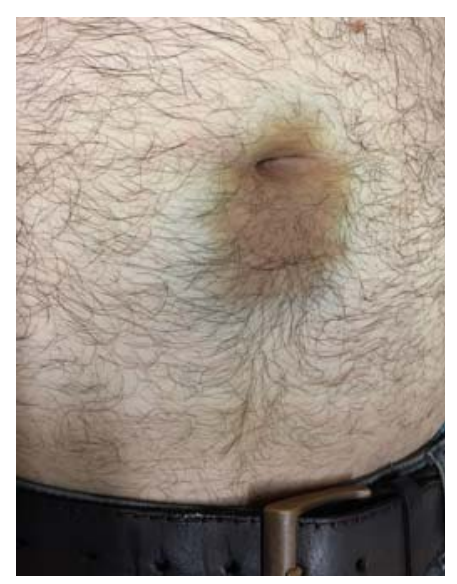

Dr. med. Klaus Dirks, Gastroenterologie und Allgemeine Innere Medizin, Interdisziplinäre Sonographie, Rems-Murr-Klinikum Winnenden, klaus.dirks@rems-murr-kliniken.de Conflict of interest statement: The author states that he has no conflict of interest.

Cite this as: Dirks K: Cullen's sign in necrotizing pancreatitis. Dtsch Arztebl Int 2018; 115: 353. DOI: 10.3238/arztebl.2018.0353

Translated from the original German by Ethan Taub, M.D. 